\title{
A high resolution induction strip readout for wire chambers *
}

\author{
A. Kruse, T. Reichel and G. Zech \\ Universität Siegen
}

October 19, 1993

\begin{abstract}
The spatial resolution of an induction strip readout for wire chambers has been optimized. Measurements with an Fe-55 source show that the position of the positive ion cloud can be determined to a precision of $10 \mu \mathrm{m}$. For minimum ionizing tracks a resolution of about $40 \mu m$ was obtained. Two charge ratios were used to infer the position from the three strips carrying the maximum charge. The charge induction was also studied with a simulation program which provides insight in the essential ingredients of a high resolution readout.
\end{abstract}

P00019989

*Work supported by Bundesminister für Forschung und Technologie (FK 056Si79I) 


\section{Introduction}

In cylindrical wire chambers a common problem is the measurement of the track coordinates parallel to the axis. Various solutions, such as the use of stereo layers, charge division, z-by-timing or combinations of those have been applied. The latter two methods offer the advantage of avoiding mirror ambiguities, but are rather unprecise. The precision of the $\mathrm{z}$ coordinate measurement obtainable with a stereo layer is better but still rather limited.

Induction strips have been proposed for position measurements already in 1969 [1] but they are less common, because in cylindrical geometries they require the insertion of stiff foils or foam structures and create additional mechanical problems especially in large central detector systems. However they provide an excellent spatial resolution and are in this respect superior to all other methods. This is essential for some experiments [2] and probably very important for the measurement of $\mathrm{CP}$ violation in $\mathrm{B}$-meson factories. So far resolutions between $100 \mu m$ and $200 \mu m$ have been reported [2,3,4], depending on the size of the detector and the method used for the position evaluation. In view of possible applications at $\mathrm{B}$-factories we have tried to optimize the resolution of an induction strip readout. From our measurements we are convinced that resolutions of better than $30 \mu \mathrm{m}$ are possible for minimum ionizing tracks.

Another field where the induction readout is of interest is synchrotron radiation. The direction of photons can be measured through the detection of a charge cloud produced by the photoelectric effect in pressurized high noble gases of high atomic number. Charge induction on strips or pads is foreseen in detectors under development [5].

In this paper we describe measurements performed with a small test chamber, which was irradiated with a Fe-55 source and cosmic muons, respectively. In section 2 we study the principle of the method by simulating the charge induction, in section 3 we present the experimental setup and the measurement, section 4, finally, summarizes the conclusions.

\section{Simulation of the induced charges}

We are interested in the position of tracks in the direction parallel to the wires ( $x$ coordinate in our notation). It is inferred from the $x$ position of the positive ions created in the avalange. Experimentally it is easier to measure the position resolution for a positive ion cloud in the direction perpendicular to the wire in the symmetry plane (y coordinate). The precision obtained experimentally for the latter can then be applied to the former. Simulations show that both resolutions should be equal within ten percent.

The signals observed at the anode wires and cathode strips of wire chambers are induced predominantly by the migration of the positive ions created in the charge avalange at distances of a few microns from the wire. For a moderate gas amplification, the positive ions are well localized on one side of the wire. This has first been observed in $1971[6]$ and has subsequently been studied experimentally and theoretically $[7,8,9]$. 
The induced charge distribution on cathode layers has been studied in the past $[10,11,12]$. The authors find similar distributions in the directions parallel and perpendicular to the wire.

For the position measurement two methods have been proposed: to identify the position with the center of gravity of the induced charge [1] and to infer it from two charge ratios [3] of three adjacent strips.

To get a good position resolution for ionizing tracks it is essential, firstly, to get a signal which is large compared to the electronic noise and, secondly, to optimize the sensitivity of the charge distribution induced on the cathode strips to the position of the positive ion cloud. In the following we discuss these two objectives.

For the computations we used the following simplified picture: We considered a single pointlike positive ion cloud produced at time zero at the surface of an anode wire and traveling without diffusion along an electric field line. The simulated detector consisted of one anode wire of $20 \mu \mathrm{m}$ diameter placed between two infinitely extended cathode planes, $5 \mathrm{~mm}$ apart. Strips were oriented either parallel or perpendicular to the wire. A charge integration time of $60 \mathrm{~ns}$ was chosen, assuming that the electrodes were coupled with low resistivity to the readout. An electric field strength $E_{0}$ of $100 \mathrm{kV} / \mathrm{cm}$ at the wire surface and a mobility of the positive ions of $2 \mathrm{~cm}^{2} / \mathrm{s} / V$ were assumed.

Of course the real world situation is much more complicated, especially when ionizing tracks are considered, but our simple model should be adequate to investigate the influence of chamber parameters on the spatial resolution.

For the numerical solution of the electrostatics we have extended an existing computer program [13] from two to three dimensions.

First we investigated the influence of the wire diameter on the charge induced on the cathode. For a fixed distance of the ion cloud from the anode wire, the fraction of the ion charges compensated on the anode wire, which one would like to keep low, increases with the wire diameter. On the other hand the distance reached during the integration time is larger for a thick wire, where the field decreases less with distance. The two effects partially compensate. The calculations show that in the case of a $20 \mu \mathrm{m}$ diameter anode wire and an integration time of $60 \mathrm{~ns}$ a fraction of 0.28 of the ion charge is induced on the cathode planes. This number does not change drastically with the diameter of the wire. It is only ten percent higher for a $10 \mu \mathrm{m}$ diameter wire and correspondingly lower for a $30 \mu m$ wire.

The partitioning of the charge on the two cathode planes depends on the position of the cloud. If the cloud is located exactly above the anode wire, 60 percent of the charge is induced on the upper cathode. This number increases slightly with the radius of the anode wire due to the larger shielding effect.

The lateral distribution of the charge induced at the cathode is shown in figure 1. It is slightly wider for the direction $(x)$ parallel to the wire than for the coordinate $(y)$ perpendicular to it. The shape of the distribution depends also on the $z$ position of the ion cloud. Thus for each projection ( $\mathrm{x}$ or $\mathrm{y}$ ) at least three strips have to be read out to measure also $z$ and to correct for the $z$ dependence. In our analysis we use only three strips, the ones that carry the largest charges, to minimize the electronic noise. We 
name them left $(L)$, central $(C)$ and right $(R)$. The total charge carries no information on the position and is eliminated by using the two charge ratios $R_{L}=Q_{L} / Q_{C}$ and $R_{R}=Q_{R} / Q_{C}$ [3]. The width of the strips has to be chosen such as to cover the part of the charge distribution (see figure 1) where is varies most and it should be kept narrow to avoid overlapping distributions from several particles. We chose the strip width equal to the distance of the wire from the cathode plane. In this way about sixty percent of the charge induced on the cathode is collected.

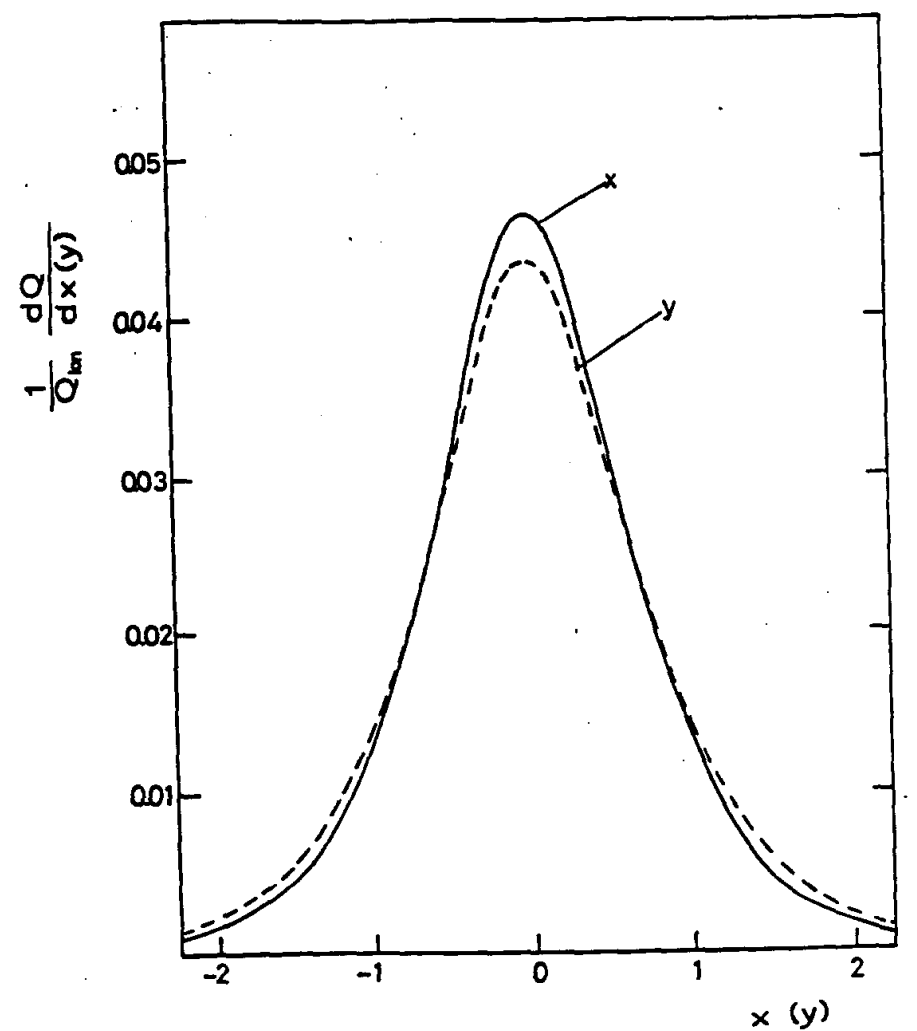

Figure 1: Spatial charge distribution induced on the cathode, projected onto the coordinates parallel $(y)$ and perpendicular $(x)$ to the wire. The coordinates are in units of half the gap. The charge is normalized to the charge of the ion cloud.

Figure 2 shows the total charge induced on the cathode planes as a function of the lateral distance $y$ of the charge cloud from the center of the anode wire. In our model chamber about twenty percent of the total charge is induced at one cathode plane after the ions have traveled $100 \mu \mathrm{m}$.

Figure 2 also shows the ratios $R_{L}$ and $R_{R}$ as a function of $\mathrm{y}$ for the case where the central strip is located exactly above the wire. A fraction of the positive ion charge is compensated by electrons located at the anode and give rise to a dipole field which is superposed to the monopole field of the remaining positive charges. The two field components induce charge ratios of opposite sign and thus partially compensate each other. The dipole contribution drops drastically with y. At distances of about $30 \mu m$ 
from the wire center the cathode charge is predominantly due to the monopole component and for larger distances the ratios rise roughly linearly with $y$.

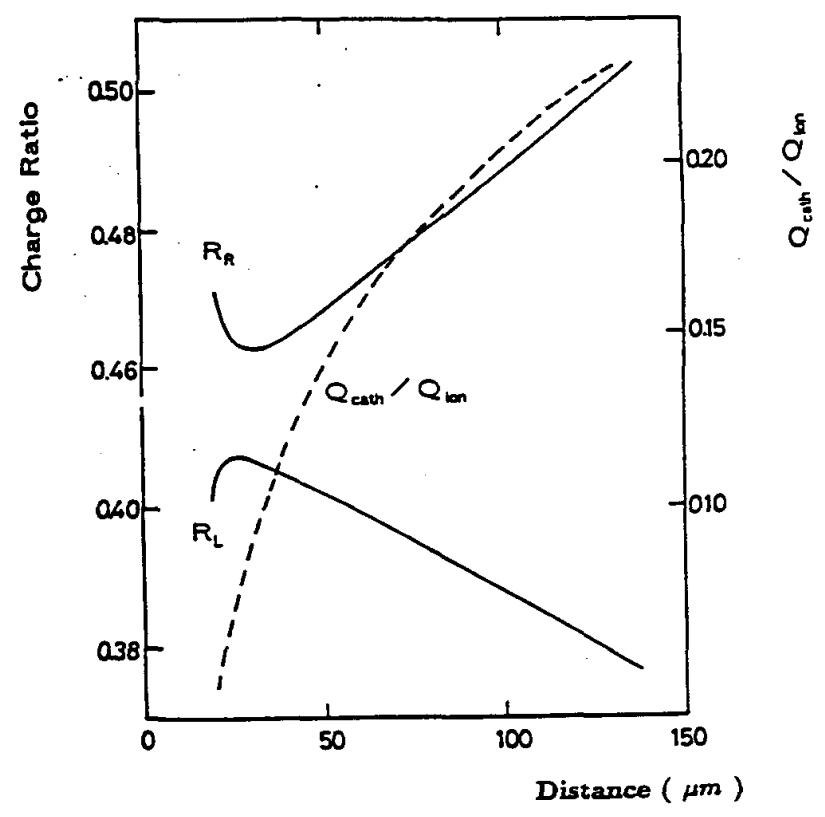

Figure 2: Fraction of the charge induced on the upper cathode (right hand scale) and strip charge ratios (left hand scale) as a function of the distance of the positive ion cloud from the anode wire. The ion cloud is localized in the symmetry plane. For the definitions of $R_{L}$ and $R_{R}$ see text.

Note, that the extrapolations of the linear part of $R_{L}$ and $R_{R}$ to $\mathrm{y}=0$ do not meet at the wire center. As a consequence of this behavior the $y$ position of the charge cloud is not identical to the center of gravity of the induced charge distribution, the difference between the two being about $40 \mu \mathrm{m}$. This effect is reduced for inclined strips and absent for strips perpendicular to the anode wire. But even then the position cannot be determined from the center of gravity or from a simple asymmetry, because these variables are not only depending on $\mathrm{x}$ but also on $\mathrm{y}$ and $z$.

From the distributions of figure 1 we can deduce the spatial resolutions of $x$ and $y$ for a known precision of the charge measurement for the strips. In the symmetric case $R_{L}=R_{R}$ and an error of one percent of the total induced charge, on each of the three strips leads to resolutions $\Delta x$ about equal $\Delta y$ of $10 \mu m$. This value changes little with the relative position of the strips with respect to the cloud. As a consequence the electronic chains of all strips have to be calibrated at least to one percent, if a resolution of $10 \mu m$ is aimed for.

For the $y$ measurement there is an additional contribution to the error from variations in the integration time. However it is negligibly small, i.e. $3 \mu \mathrm{m}$ for a $10 \mathrm{~ns}$ variation in the gate length.

We can summarize our findings as follows: The diameter of the anode wire has little 

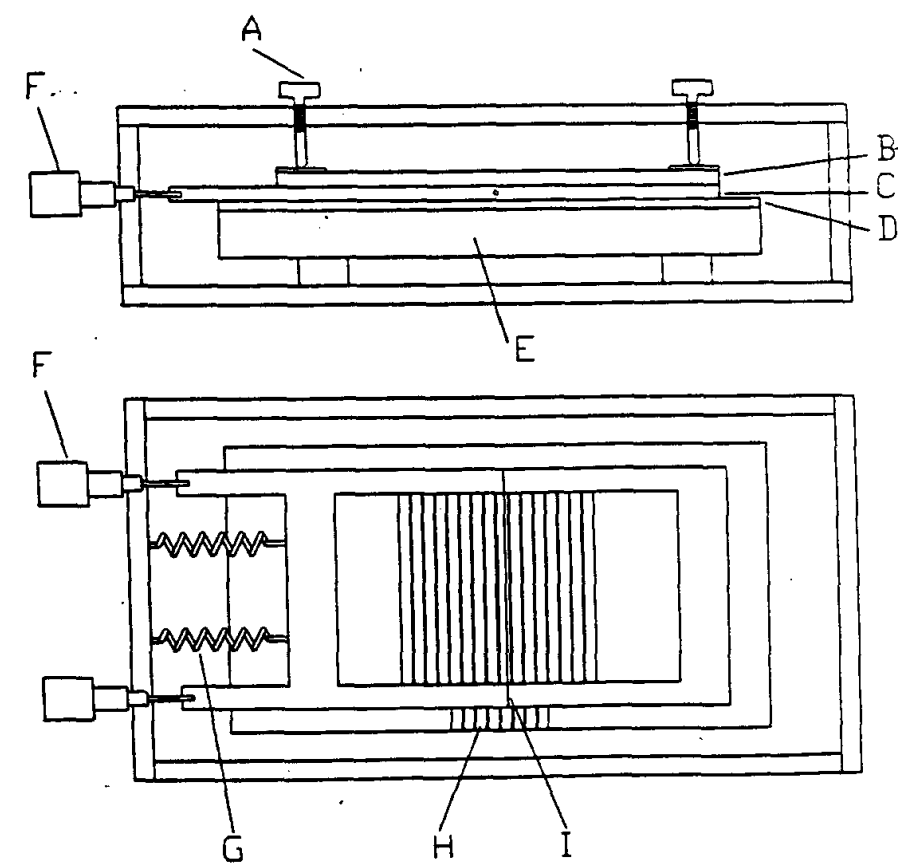

Figure 3: Schematic sketch of the test chamber. The components are the wire frame $(C)$, the cathode frames $(B, D)$, fixation screws $(A)$, micrometer drives $(F)$, restoring springs (G) and anode wire (I).

influence on the resolution, smaller diameters being slightly more favorable. To obtain resolutions of about $10 \mu \mathrm{m}$, three strips leading to two variables (charge ratios) have to be used. Assuming a constant error of the charge measurement, the charge of the central strip has to be measured to one percent. Timing is no problem for integration times of about $60 \mathrm{~ns}$. Very short integration times below $20 \mathrm{~ns}$ should be avoided, because of the smallness of the signal, the wide charge distribution on the cathode and the more critical timing. Of course the errors on the coordinates scale with the gap between anode and cathode if the ratio strip width to gap is kept constant. A small width has the additional advantage of good double hit separation but obviously has to be paid for by a higher mechanical complexity and an increased number of readout channels.

\section{The measurements}

To check the results of the previous section we have built the simple one-wire-chamber shown in figure 3. The wire was oriented parallel to the cathode strips and could be moved with respect to the strips and adjusted by two screws to a precision of one micron. The anode wire had a diameter of $20 \mu \mathrm{m}$ and was located in the center between the two cathode planes. The distance to the planes was $2.5 \mathrm{~mm}$, equal to the pitch of the cathode strips. The strips were $2.3 \mathrm{~mm}$ wide and $0.2 \mathrm{~mm}$ apart from each other. We used a gas mixture of 90 parts argone and 10 parts methane and operated the chamber at $1.35 \mathrm{kV}$.

The cathode signals were read out with standard charge sensitive amplifiers and shapers similar to those used for the drift chambers of the ZEUS detector. The amplified signals were digitized and recorded in 12 bit CAMAC ADCs. The integration gate was derived from the anode wire. All channels were carefully calibrated, comparing the pulse 


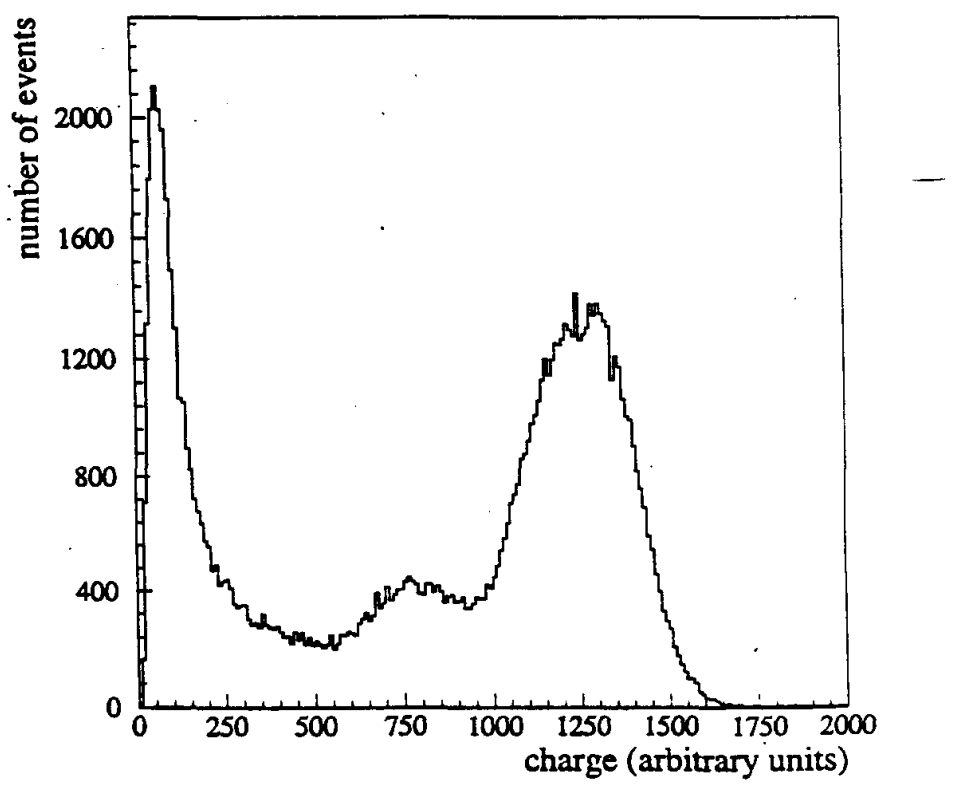

Figure 4: Pulse height spectrum from the Fe-55 source.

heights from an Fe-55 source. The pulse height distribution deduced from the three strips with the largest signals is presented in figure 4 .

During the integration time the positive ions reach a distance of about $100 \mu \mathrm{m}$. The lateral spread of the ion cloud is a few microns only, with an exponentially decreasing tail to larger distances. Thus the events are expected to correspond to a circular distribution in the $z-y$ projection. However positions near the symmetry plane $z=0$ are expected to be much more abundant than the other orientations: Because of the flat chamber geometry most of the primary electrons arrive horizontally at the anode and correspondingly the positive ions travel the same way back.

This is visualized in figure 5 which is a surface plot of the event rate as a function of $R_{L}$ versus $R_{R}$. The two sharp peaks of the crown shaped distribution correspond to positions left and right from the wire in the horizontal plane.

Figure 5 gives a qualitative impression of the position resolution since the spatial distance of the ion clouds associated to the peaks is about $200 \mu m$ and the f.w.h.m. of the peaks is about ten percent of this number, i.e. around $20 \mu \mathrm{m}$. For a quantitative estimate the variables $R_{L}$ and $R_{R}$ have to be transformed into the coordinates $\mathrm{y}$ and $z$. The transformation can be computed from the simulation. We have preferred to deduce the transformation experimentally, moving the anode wire. As an example, we show in figure 6 the crowns obtained from three wire positions shifted by $100 \mu m$. The first and the fourth peak correspond to the same crown.

Figure 7 is a histogram of the $y$-position for events, where the ion cloud was located near the $x-y$ plane $( \pm 5 \mu \mathrm{m})$. Qualitatively it is a cross section through the left hand peak of the crown of figure 5 . The distribution has practically no tails and corresponds to a r.m.s. resolution of $9 \mu \mathrm{m}$. A resolution of $35 \mu \mathrm{m}$ has been reported [14] in a previous measurement.

The precision which one could obtain in the $\mathrm{x}$ direction with strips perpendicular to 


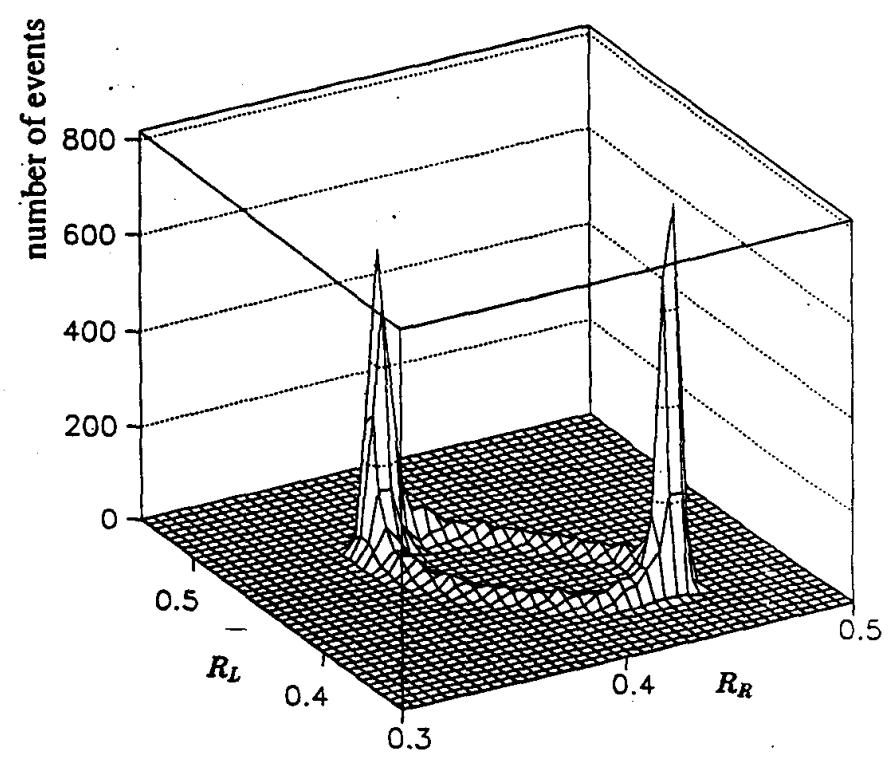

Figure 5: Surface plot of the event rate as a function of the charge ratios for Fe-55 events. The two peaks correspond to positive ion clouds located in the symmetry plane at $100 \mu \mathrm{m}$ distance from the anode wire.

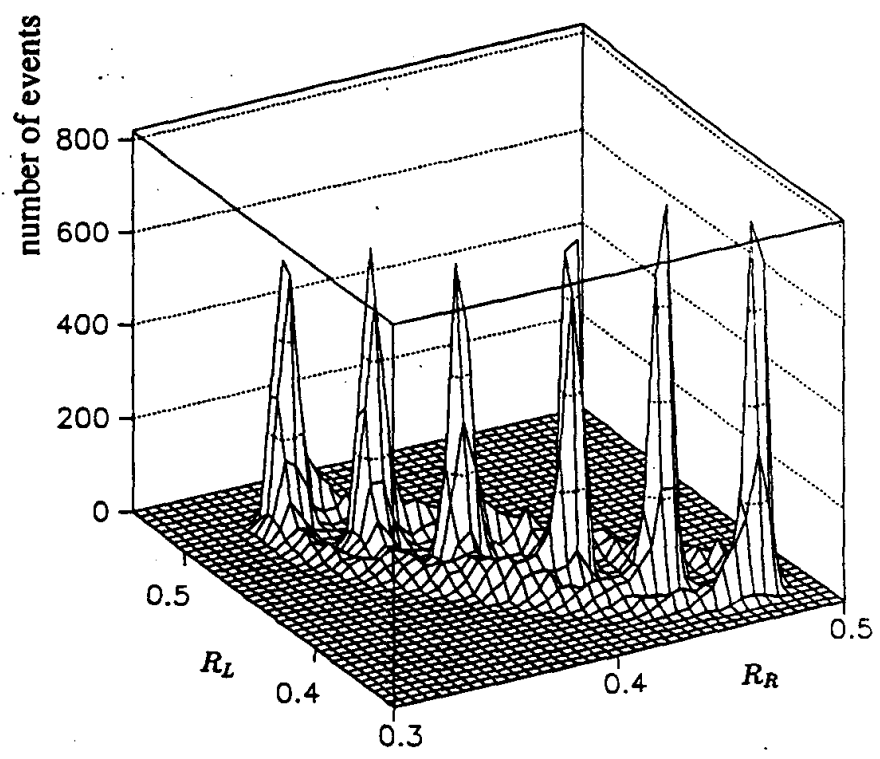

Figure 6: Surface plot of the event rate as a function of the charge ratios for Fe-55 events. The distributions for three wire positions, each shifted by $100 \mu \mathrm{m}$ are superposed. 


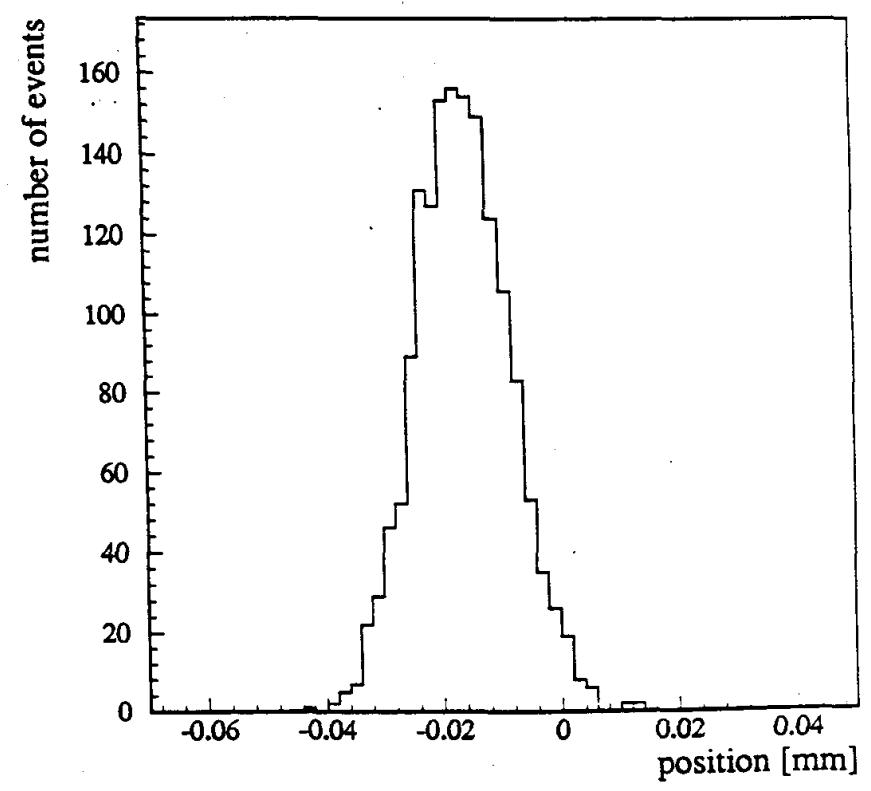

Figure 7: Spatial distribution of ion clouds for Fe-55 events. The width of the peak corresponds to a r.m.s. resolution of $9 \mu \mathrm{m}$.

the anode wire is harder to measure. However our calculations show that it should be even slightly better than in $\mathrm{y}$. We have also determined the $\mathrm{z}$ position resolution for the ionization cluster from our crown and find a value of better than $15 \mu \mathrm{m}$.

When we compared the experimental results with the simulation, we realized that the wire was slightly off center in the $z$ direction. From the measurements the wire position in both directions could be determined to better than one micron. In principle, also in large chambers the positions of anode wires can be calibrated relative to induction strips with an $\mathrm{Fe}-55$ source to the same precision.

We repeated our measurements with cosmic ray muons after adjusting the gas amplification to the lower primary ionization. The events were triggered by a $1 \mathrm{~ms}$ long gate derived from two scintillators. Noise was rejected by a pulse height cut. We selected relatively large drift times between $100 \mathrm{~ns}$ and $500 \mathrm{~ns}$ to enhance events in the horizontal plane.

As expected, the resolution is less brilliant than for the photon clusters, because now the induced charges produced by several primary clusters overlap. Also the total charge for a minimizing track is significantly lower than that from a Fe-5.5 photon. In the scatter plot of $R_{L}$ versus $R_{R}$, shown in figure 8, the crown observed for photon events is no longer visible but as a remanent of the peaks two clusters are present.

The same procedure as described above, leads to the spatial distribution of figure 9 . The r.m.s resolution is $40 \mu m$. We expect that the resolution would improve significantly in a test beam with clean, perpendicular tracks. 


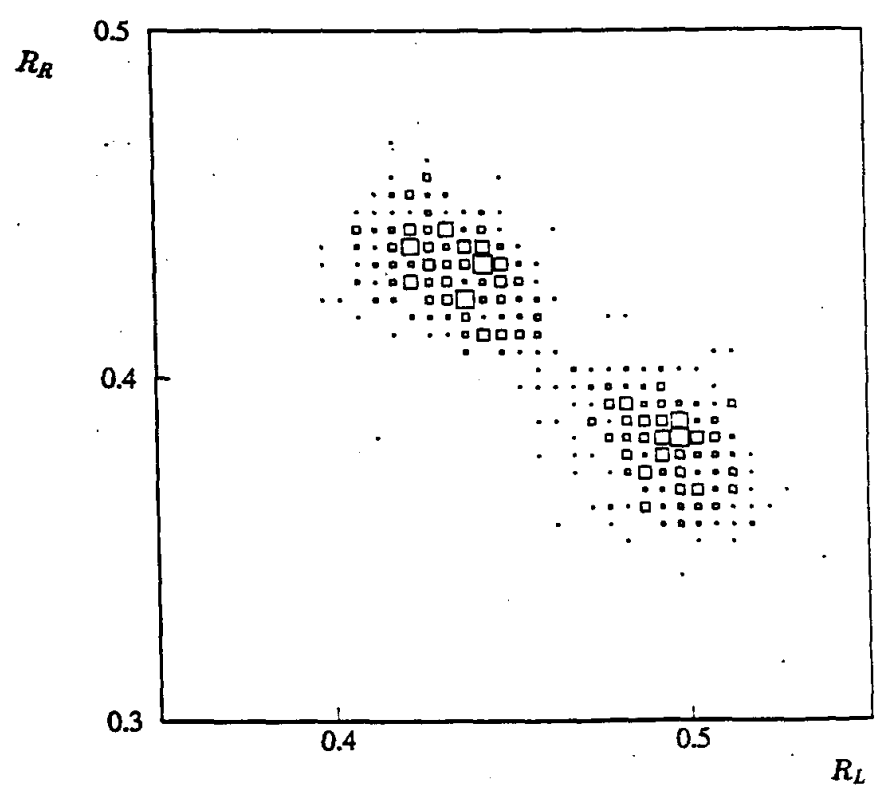

Figure 8: Scatter plot of the event rate as a function of the charge ratios for cosmic muons.

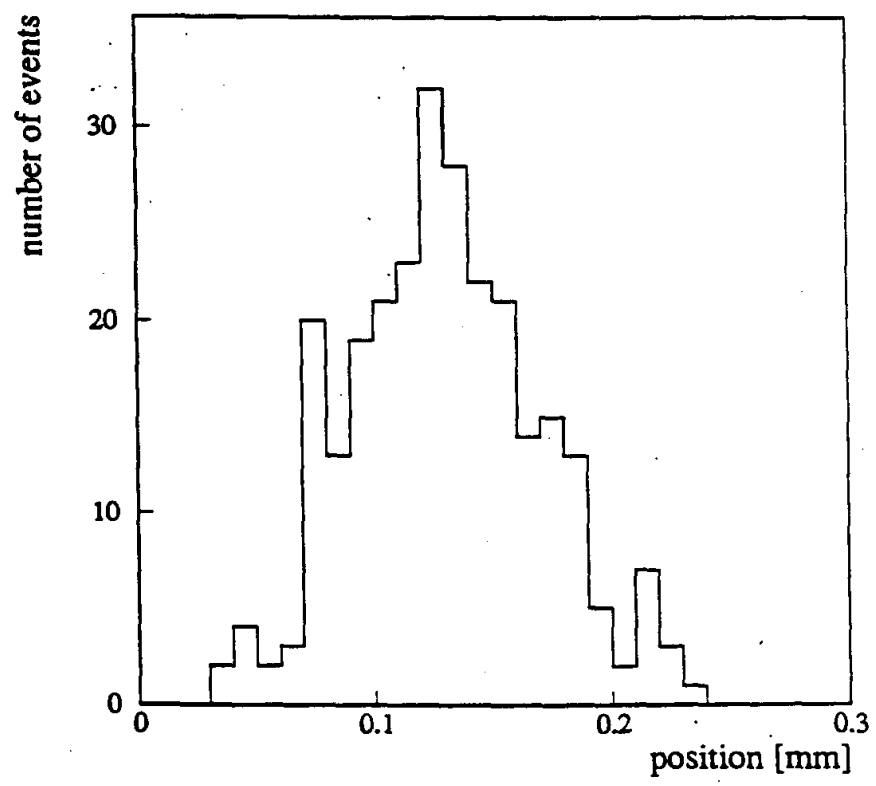

Figure 9: Spatial distribution for cosmic muons. The width of the peak corresponds to a r.m.s. resolution of $40 \mu \mathrm{m}$. 


\section{Summary and conclusions}

The position of a positive ion cloud, produced by gas amplification on an anode wire can be localized to $10 \mu \mathrm{m}$ with induction strips. This has been demonstrated for the coordinate perpendicular to the wire with a simple test setup containing one wire and induction strips of $2.5 \mathrm{~mm}$ pitch irradiated by an Fe-55 source. Numerical calculations confirm this result and predict an even better resolution for the coordinate parallel to the wire. This precision is sensitive to the strip pitch which should roughly equal the distance of the cathode plane from the wire and relies on a precise calibration of the electronic channels at a level of a percent. Small diameters of the anode wires are favorable.

For minimum ionizing tracks traversing a chamber perpendicularly a spatial resolution of better than $30 \mu \mathrm{m}$ is expected for the coordinate parallel to the wire. Using cosmic muons we have obtained $40 \mu m$ for the coordinate perpendicular to it.

To achieve a resolution of the order of a few tens of micrometers, the geometry of the detector has to be known to a very high precision. For the lithographic production of induction strips this is trivial. The gap height and the positions of the anode wires can be calibrated by tracks or more easily by $\gamma$-sources. We have checked this with a small detector.

To obtain a good precision it is mandatory to use a two parameter method - like the charge ratio method - for the reconstruction. A simple center of gravity parametrization cannot provide a good resolution. Especially when the induction strips are not oriented perpendicular to the anode wires its results are strongly biased. 


\section{References}

[1] G. Charpak, D. Rahm and H. Steiner, Nucl. Instr. and Meth. 80 (1970) 13

[2] Breskin et al., Nucl. Instr. and Meth. 143 (1977) 29

[3] J. Chiba et al., Nucl. Instr. and Meth. 206 (1983) 451

[4] M. Dejardin et al., Nucl. Instr. and Meth A283 (1989) 484

[5] Proceedings of the European Workshop on X-Ray Detectors for Synchrotron Radiation Sources, Aussois, ed. A.H. Walenta (1991)

[6] A. H. Walenta, J. Heintze and B. Schürlein, Nucl. Instr. and Meth. 92 (1971), 373

[7] J. Fischer, H. Okuno and A. H. Walenta, Nucl. Instr. and Meth. 151 (1978), 451

[8] H. Okuno J. Fischer, V. Radeka and A. H. Walenta, IEEE Trans. Nucl. Sci. NS-26 (1979) 160

[9] K. Urase et al., Nucl. Instr. and Meth. A290 (1990) 401

[10] J. S. Gordon, E. Mathieson, Nucl. Instr. and Meth. 227 (1984) 267

[11] J. R. Thompson, J. S. Gordon, E. Mathieson, Nucl. Instr. and Meth. A234 (1985) 505

[12] H. v. d. Graaf and J. P. Wagenaar, Nucl. Instr. and Meth. A252 (1986) 311

[13] W. Weihs and G. Zech, Nucl. Instr. and Meth. A281 (1989)

[14] G. Charpak, G. Peterson, A. Policarpo and F. Sauli, Nucl. Instr. and Meth. 148 (1978) 471 